\title{
Hipertensão Arterial Sistêmica: aspectos clínicos e análise farmacológica no tratamento dos pacientes de um setor de Psicogeriatria do Instituto Bairral de Psiquiatria, no Município de Itapira, SP
}

\author{
Systemic Arterial Hypertension: clinical aspects and pharmacological analysis in \\ the treatment of patients of a Psychogeriatrics Sector of Bairral Psychiatry \\ Institute, Itapira Municipality, São Paulo State, Brazil

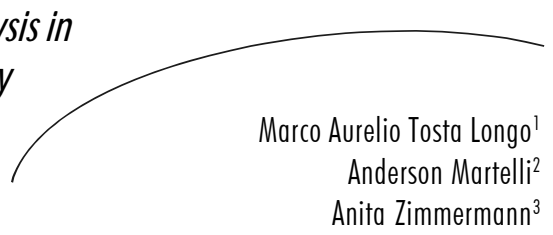

Objetivo: o presente trabalho teve como objetivo a verificação dos quadros de hipertensão arterial sistêmica (HAS) e análise das principais classes dos agentes anti-hipertensivos orais usados pelos pacientes integrantes da Unidade Terapêutica - Setor Estância do Instituto Bairral de Psiquiatria localizado na cidade de Itapira, SP. Metodologia: a população analisada foi composta por 51 sujeitos com faixa etária entre 52 e 87 anos de idade, em sua maioria portadora de distúrbios psiquiátricos e outras comorbidades clínicas, sendo excluídos da amostra os pacientes sem histórico de HAS e com níveis pressóricos considerados normais pelas $V$ Diretrizes Brasileiras de Hipertensão Arterial. O material para realização desta pesquisa foi obtido a partir da revisão bibliográfica de livros e artigos científicos publicados em periódicos localizados nas bases de dados Medline, Scielo e Lilacs, tendo como palavras-chave: hipertensão arterial sistêmica; anti-hipertensivos; idoso. Trata-se de um estudo transversal com dados obtidos a partir de pesquisa em prontuários de anotações técnicas, para a caracterização dos quadros diagnosticados como hipertensão arterial, considerando anamnese, exame físico na admissão e controle dos níveis pressóricos diários dos pacientes da Unidade Terapêutica Estância do Instituto Bairral de Psiquiatria, entre os dias 18 a 25 de setembro de 2009, com posterior análise das principais classes dos agentes anti-hipertensivos orais utilizados. Resultados: dos 51 pacientes analisados, 27 compuseram a amostra, pois foram diagnosticados com quadro de hipertensão arterial no período referente, correspondendo a $53 \%$ do número total, havendo predominância do sexo feminino em 15 casos (56\%). Quanto à frequência dos fármacos anti-hipertensivos prescritos na amostra, observou-se que os inibidores da enzima conversora de angiotensina (IECA) foi o grupo de medicamentos mais utilizado em especial o captopril,

\footnotetext{
Faculdade de Ciências Médicas, Pós-graduação em Geriatria. Universidade Estadual de CampinasUnicamp, Campinas, SP, Brasil.

2 Faculdade de Ciências Médicas, Pós-graduação em Laboratório Clínico (Patologia Clínica). Universidade Estadual de Campinas-Unicamp, Campinas, SP, Brasil.

3 Faculdade de Ciências Médicas, Pós-graduação em Ciências Médicas, Departamento de Oftalmo/ Otorrinolaringologia. Universidade Estadual de Campinas-Unicamp,Campinas, SP, Brasil.
}

Palavras-chave:

Farmacologia. Saúde do idoso. Observação clínica. Hipertensão arterial sistêmica. Antihipertensivos. Idoso. 
seguido pelo grupo dos diuréticos tiazídicos. Em associação, os IECA, juntamente com os diuréticos, foram os mais prescritos. Conclusão: este estudo demonstrou ser significativo o número da população idosa com HAS no Setor Estância do Instituto Bairral de Psiquiatria, sugerindo acompanhamento clínico e medicamentoso dessa clientela. A terapêutica medicamentosa adotada para tratamento da HAS no setor em questão está de acordo com a recomendada na literatura.

\section{Abstract}

Objective: This study aimed to check the tables of Hypertension Blood Pressure (HBP) and analysis of the major classes of oral antihypertensive agents used by patients members of the Therapeutic Unit - Bairral Industry office of the Institute of Psychiatry located at Itapira, SP. Methodology: The study sample consisted of 51 subjects aged between 52 and 87 years old, mostly suffering from psychiatric disorders and other clinical morbidities, being excluded from the sample patients with no history of hypertension and those whose blood pressure levels are considered normal by V Brazilian Guidelines on Hypertension. The material for this research was obtained from the literature review of books and scientific papers published in journals located in Medline, Lilacs and Scielo, with the key words: hypertension, antihypertensive; elderly. This is a cross-sectional study with data obtained from research records of technical notes for the characterization of the tables diagnosed with hypertension, considering anamnesis, physical examination on admission and daily control of blood pressure levels of patients in the Unit Office of Bairral Therapeutics Institute of Psychiatry, from the 18th to 25th September 2009, with subsequent analysis of the major classes of oral antihypertensive agents used. Results: Of the 51 patients studied, 27 were included in the study because they were diagnosed with hypertension in the regarded period, accounting for $53 \%$ of the total number and having female predominance in 15 cases (56\%). As the frequency of antihypertensive drugs prescribed in the sample, it was observed that inhibitors of angiotensin converting enzyme (ACE) was the most widely used group of medicines especially captopril, followed by the group of thiazide diuretics. Concomitantly, ACE together with diuretics were the most frequently prescribed. Conclusion: This study demonstrated a significant number of elderly people with hypertension in the Bairral sector office of the Institute of Psychiatry, suggesting clinical and medical care of this clientele. Drug therapy of hypertension treatment adopted for the sector in question is consistent with the recommended literature.
Key words: Pharmacology . Health of the Elderly. Clinical Symptoms Hypertension blood pressure. Antihypertensive. Elderly.

\section{INTRODUÇÃO}

No Brasil, as doenças cardiovasculares são responsáveis por mais de 250.000 mortes por ano, e a hipertensão arterial sistêmica (HAS) participa de quase metade delas. ${ }^{1}$ Estudos recentes mostram que, entre os idosos, sua prevalência varia de $52 \%$ a $63 \%{ }^{2-6}$ o que permite identificar a HAS como um problema de saúde pública, conferindo ao paciente um alto risco cardiovascular. ${ }^{7,8}$
Com o envelhecimento fisiológico, ocorre o desenvolvimento de processos ateroscleróticos nos grandes vasos e arteríolas, ocasionando perda da distensibilidade e elasticidade, diminuindo sua capacidade com o aumento da velocidade da onda de pulso. A rigidez da parede dos vasos tende a elevar a pressão sistólica e o aumento da velocidade da onda de pulso mantém a pressão arterial diastólica (PAD) dentro dos valores normais ou pode até diminuí-la. Com isso, a pressão sistólica e a pressão de pulso têm-se definido como 
adequados preditores de eventos cardiovasculares no idoso. ${ }^{9}$

Ensaios clínicos de tratamento da HAS no idoso realizadas na última década comprovam a necessidade do controle pressórico como forma de redução do risco cardiovascular, tanto no caso de hipertensão sisto-diastólica ou hipertensão sistólica isolada. ${ }^{1}$

Uma revisão de oito ensaios incluindo mais de 15.000 pacientes com 60 anos ou mais mostrou que o uso de medicação anti-hipertensiva reduz em $13 \%$ a mortalidade, $30 \%$ o acidente vascular cerebral e $23 \%$ os eventos coronarianos. ${ }^{10}$

O ensaio clínico HYVET (Hipertension in the Very Elderly Trial), envolvendo 3.845 pacientes com mais de 80 anos com pressão sistólica maior ou igual a $160 \mathrm{mmhg}$, mostrou em dois anos redução nos eventos fatais de acidente vascular cerebral (AVC) de 10,7\% no grupo placebo, para 6,5\% no grupo com tratamento ativo. A morte por qualquer causa foi reduzida de 59,6\% no grupo placebo para $47,2 \%$ no grupo com tratamento. ${ }^{9}$

Infelizmente, dados provenientes de alguns estudos sugerem que $30 \%$ a $50 \%$ dos pacientes hipertensos, mesmo em tratamento medicamentoso, não apresentam pressão arterial controlada $^{7,11}$, e em cerca de $10 \%$ dos idosos, o diagnóstico de HAS somente é feito após um evento clínico decorrente da pressão elevada por vários anos. ${ }^{1}$

No idoso, peculiaridades devem ser consideradas durante a aferição da pressão arterial e diagnóstico da HAS, podendo ocorrer a pseudohipertensão, hiato auscultatório, hipotensão pósprandial, hipertensão do avental branco e hipotensão ortostática. ${ }^{9,10}$

A maior parte dos pacientes com hipertensão apresenta excesso de peso, e estudos de diferentes populações mostram que o sobrepeso e a obesidade podem ser responsáveis por $20 \%$ a $30 \%$ dos casos de hipertensão arterial. ${ }^{12}$ Estudos clínicos no tratamento da pressão arterial sugerem modificações do estilo de vida com a realização de atividades físicas e redução do peso corporal como primeiro passo na redução da pressão arterial ${ }^{1,11,13}$, restrição do sal na dieta, associado ou não ao uso de medicamentos que podem também ser administrados isolados ou em associação. ${ }^{1,11,14,15}$

O presente trabalho teve como objetivo verificar os quadros de hipertensão arterial sistêmica e análise das principais classes dos agentes anti-hipertensivos orais usados pelos pacientes integrantes da Unidade Terapêutica - Setor Estância do Instituto Bairral de Psiquiatria, localizado na cidade de Itapira.

Por se tratar de uma unidade compreendida por pacientes com faixa etária avançada (senil e pré-senil), apresentam maior probabilidade de desenvolvimento da HAS, sendo considerado um setor ideal para a pesquisa em questão.

\section{CLASSE DOS PRINCIPAIS MEDICAMENTOS ANTI- HIPERTENSIVOS}

Diversas classes de anti-hipertensivos já demonstraram reduzir o risco cardiovascular e, na maioria dos casos, torna-se necessário associar fármacos com mecanismos de ação diferentes. Além da evidência de benefício clínico, a escolha do anti-hipertensivo deve considerar fatores como comorbidades do paciente, o perfil de efeitos adversos, a interação medicamentosa, a posologia e até mesmo o preço do fármaco no mercado. ${ }^{11,16}$

Diferentemente dos jovens, os idosos possuem em média três a cinco doenças crônicas associadas e somente 6\% são considerados livres de doenças. As comorbidades frequentemente norteiam a escolha do anti-hipertensivo, devendo-se dar preferência aos fármacos que também possam trazer benefícios às outras doenças existentes. ${ }^{1,10,16}$

Nos idosos, deve-se também avaliar as particularidades no tratamento medicamentoso vistas nas alterações fisiológicas próprias do envelhecimento, como diminuição da atividade dos barorreceptores, alterações da composição corpórea, do metabolismo basal, do fluxo 
sanguíneo hepático e do ritmo de filtração glomerular, com alteração da absorção, da distribuição e metabolização dos medicamentos. ${ }^{17}$

A introdução do anti-hipertensivo deve ser feita em doses baixas e com aumento gradual, evitando-se quedas tensionais que possam acarretar hipotensão ortostática ou hipofluxo em órgãos vitais. Octogenários merecem atenção especial, pois tendem a responder de maneira inesperada à terapia medicamentosa. ${ }^{9}$ Os pacientes devem ser orientados em relação à doença durante as consultas médicas e, sempre que possível, em grupos de assistência multiprofissional..$^{1,16}$

A seguir, as principais classes de medicamentos anti-hipertensivos:

\section{Bloqueadores adrenérgicos (Grupo I)}

São drogas que intervêm na transmissão simpática. A maioria dos antagonistas de receptores adrenérgicos é seletiva para os receptores $\alpha$ ou $\beta$, e muitos também são seletivos para seus subtipos. $^{18,19}$

Os antagonistas dos receptores $\beta$-adrenérgicos constituem um grupo importante de fármacos. Foram descobertos em 1958, dez anos após Ahlquist ter postulado a existência dos receptores $\beta$-adrenérgicos. Avanços nesta área conduziram ao propranolol, que é um antagonista potente e bloqueia igualmente os receptores $\beta_{1}$ e $\beta_{2}{ }^{19}$

Os mecanismos de ação desta classe de drogas anti-hipertensiva são múltiplos, incluindo: diminuição do débito cardíaco, efeitos centrais, readaptação dos barorreceptores, diminuição da liberação de renina e inibição simpática periférica. ${ }^{20}$

Como efeito adicional, é importante ressaltar suas propriedades antiarrítmicas e antianginosas, frequentemente úteis em pacientes hipertensos portadores de insuficiência cardíaca, insuficiência coronariana, angina e infarto agudo do miocárdio. Não são indicados como monoterapia inicial em idosos sem comorbidades, visto que falharam em mostrar benefícios cardioprotetores nesta população. . $^{1,20}$
Pode-se citar como exemplos o atenolol, doxazosina e propranolol..$^{18,19}$

\section{Bloqueadores dos canais de cálcio (Grupo II)}

Os antagonistas do cálcio terapeuticamente importantes atuam sobre os canais do tipo L, compreendendo, assim, três classes quimicamente distintas: fenilalquilaminas (verapamil), benzotiazepinas (diltiazem) e diidropiridinas (nifedipina, amlodipina). ${ }^{18,19}$

Os fármacos de cada uma dessas três classes ligam-se às subunidades á do canal de cálcio cardíaco do tipo L, mas em locais distintos e que vão interagir alostericamente entre si e com o maquinário de controle da passagem de cálcio, impedindo sua abertura e consequentemente reduzindo a entrada de cálcio. ${ }^{19}$

$\mathrm{Na}$ musculatura lisa, causam dilatação arterial/arteriolar generalizada e diminuição de sua resistência, reduzindo a pressão arterial. ${ }^{18-20}$

A nifedipina para uso sublingual, apesar de sua popularidade nas unidades de emergência de todo Brasil, inclusive em serviços ligados a escolas médicas, tem sofrido críticas severas pelos especialistas nacionais e internacionais, pois a queda da pressão arterial pode ocorrer de forma abrupta e inesperada, determinando hipofluxo em órgãos nobres com desastrosas consequências. ${ }^{20}$

\section{Diuréticos (Grupo III)}

É considerada a classe de fármacos antihipertensivos mais utilizada, em virtude da sua eficácia terapêutica e baixo custo. ${ }^{21}$ São substâncias com ação sobre os rins, atuando de forma a aumentar a taxa do débito e volume urinário, consequentemente a excreção urinária de solutos, em especial o sódio e cloreto. ${ }^{19,20}$ Seu efeito primário consiste em diminuir a reabsorção de sódio pelos túbulos, causando natriurese (maior débito de sódio), o que por sua vez, causa diurese (maior débito de água), sendo o aumento da perda de água secundário à excreção aumentada de sódio, já que o sódio remanescente nos túbulos age de forma osmótica, diminuindo a reabsorção de água. ${ }^{13,19}$ 
O uso clínico mais comum dos diuréticos é para reduzir o volume de liquido extracelular, especialmente em doenças associadas a edema e hipertensão, inibindo a reabsorção tubular em locais diferentes ao longo do néfron renal. . $^{13,21,22}$

As classes gerais de diuréticos e seus mecanismos de ação são listados a seguir.
Diuréticos de alça - a furosemida, ácido etacrínico e bumetamida são diuréticos potentes que diminuem a reabsorção ativa no segmento ascendente espesso da alça de Henle ao bloquearem o cotransportador de 1 sódio, 2 cloretos, 1 potássio, localizado na membrana luminal das células epiteliais (fig.1). Esses diuréticos estão entre os mais potentes usados clinicamente. . $^{13,19,20}$

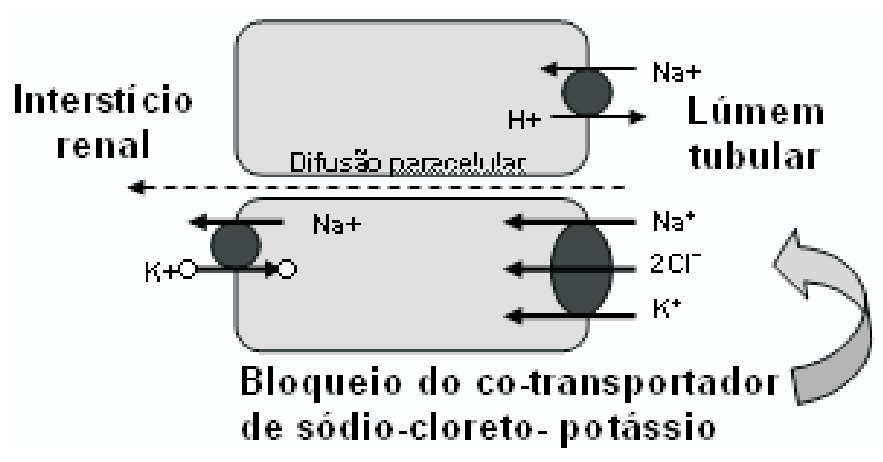

Figura 1 - Sitio de ligação dos diuréticos de alça nas células tubulares bloqueando o co-transportador de sódio-cloreto-potássio, favorecendo o débito urinário desses eletrólitos com diminuição da reabsorção de liquido. Adaptado do livro Tratado de Fisiologia Médica, 2006.

Ao bloquear o cotransporte ativo de sódio, cloreto e potássio na membrana luminal da alça de Henle, os diuréticos de alça aumentam o débito urinário desses eletrólitos e também de outros, bem como o de água. ${ }^{18,20}$

Esse mecanismo pode ocorrer por duas razões: primeiramente, eles aumentam muito as quantidades de solutos liberados para as partes distais dos néfrons, atuando como agentes osmóticos evitando a reabsorção de água, ou interrompendo o sistema multiplicador de contracorrente, diminuindo a absorção de íons da alça de Henle para o interstício medular, diminuindo assim a osmolaridade do líquido intersticial medular, comprometendo a capacidade dos rins de concentrar ou diluir a urina. A baixa osmolaridade do líquido intersticial da medula renal diminui a absorção de água pela alça descendente de Henle, produzindo um débito urinário 25 vezes acima do normal. ${ }^{13}$
Os diuréticos de alça podem ser eficazes, mesmo em pacientes com a função renal seriamente comprometida. ${ }^{20}$

Diuréticos tiazídicos - os diuréticos tiazídicos foram os primeiros anti-hipertensivos disponíveis para uso em larga escala. Lançados em meados dos anos 50, continuam a ser administrados, isolados ou em associação, a milhões de hipertensos em todo o mundo. ${ }^{23}$

Os derivados tiazídicos, como a clortalidona e hidroclorotiazida, atuam basicamente na parte proximal dos túbulos contorcidos distais, bloqueando o cotransportador de sódio-cloreto na membrana luminal das células tubulares (fig. 2)..$^{13,19,21}$ Sob condições favoráveis, esses agentes fazem com que $5 \%$ a $10 \%$ do filtrado glomerular passem para a urina. ${ }^{13}$

Os diuréticos tiazídicos apresentam uma ação vasodilatadora que ainda não está completamente elucidada, podendo causar 
também hiperglicemia. ${ }^{19} \mathrm{~A}$ queda inicial da pressão arterial decorre da diminuição da volemia causada pela diurese, mas a fase tardia também está relacionada a uma ação sobre a musculatura lisa vascular. ${ }^{14,19,24}$
Estudos revelam queo diazóxido, um tiazídico nãodiurético, apresenta potentes efeitos vasodilatadores causados pela ativação dos canais $\mathrm{K}_{\mathrm{ATP}}$ implicados no controle do potencial de membrana no músculo liso vascular e na inibição da secreção de insulina. ${ }^{19}$

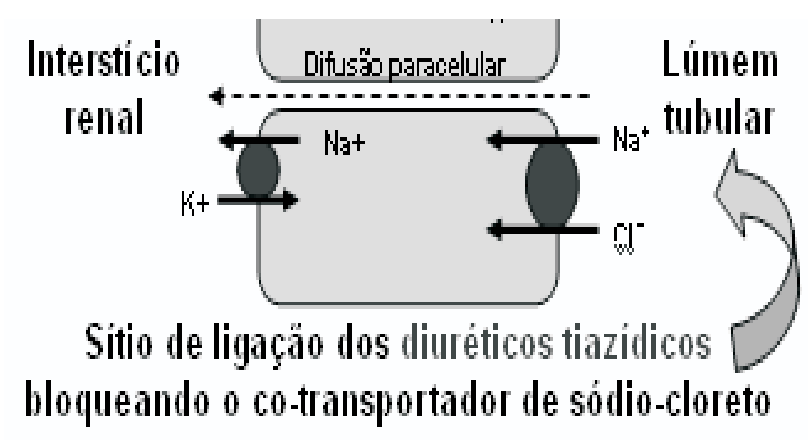

Figura 2 - Sitio de ligação dos derivados tiazídicos bloqueando o co-transportador de sódio-cloreto, favorecendo o débito urinário desses eletrólitos com diminuição da reabsorção do filtrado. Adaptado do livro Tratado de Fisiologia Médica, 2006.

Inibidores competitivos da aldosterona apresentam-se como antagonistas da aldosterona a espironolactona e esplerenona, que competem com este hormônio pelos sítios receptores nas células epiteliais do túbulo coletor cortical, reduzindo a absorção de sódio e secreção de potássio nesse segmento tubular (fig. 3). Consequentemente, o sódio permanece no túbulo agindo como diurético osmótico, causando aumento da excreção de água e sódio. ${ }^{13,19}$

Na medida em que esses fármacos bloqueiam os efeitos da aldosterona, de promover a secreção de potássio pelos túbulos, eles diminuem a excreção desse íon, aumentando sua concentração no liquido extracelular e por isso são referidos como diuréticos poupadores de potássio. ${ }^{13}$
Diuréticos que bloqueiam canais de sódio nos túbulos coletores - a amilorida é um exemplo de fármaco que inibe a reabsorção de sódio e secreção de potássio de modo semelhante à espironolactona. Entretanto, ao nível celular, agem diretamente bloqueando os canais de sódio da membrana luminal das células epiteliais do túbulo coletor (fig. 3). ${ }^{13,18,19}$

Devido a essa menor entrada de sódio nas células epiteliais do túbulo coletor, ocorre também redução no transporte desse íon pelas membranas basolaterais; consequentemente, menor atividade $\mathrm{da}$ atepase $\mathrm{Na} / \mathrm{K}$. Essa diminuição de atividade reduz o transporte de potássio para as células e, por fim, diminui a secreção de potássio para o líquido tubular. Por essa razão, os bloqueadores dos canais de sódio são também considerados diuréticos poupadores de potássio. ${ }^{13}$ 


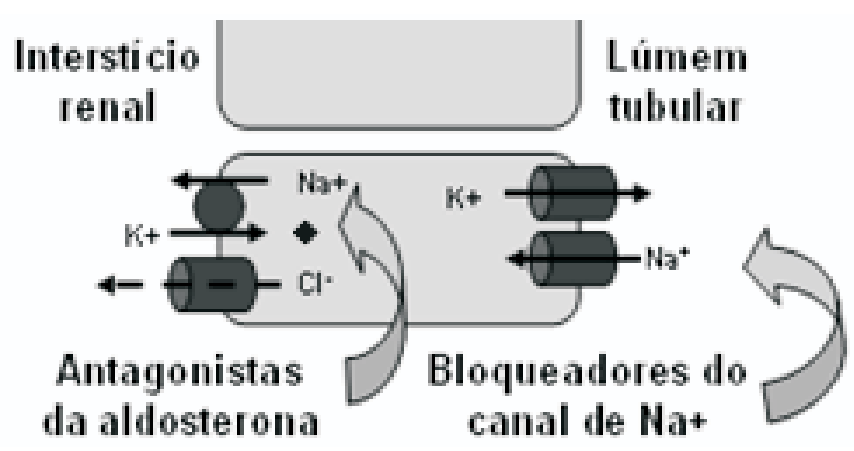

Figura 3 - Ação dos antagonistas da aldosterona competindo pelo sitio de ligação (azul) desse hormônio com redução da absorção de sódio e secreção de potássio, e na região apical a ação de bloqueadores diretos dos canais de sódio com função semelhante. Adaptado do livro Tratado de Fisiologia Médica, 2006.

Drogas que intervêm no sistema reninaangiotensina (Grupo IV)

O sistema renina-angiotensina aldosterona (SRAA) desempenha importante função na regulação da pressão arterial e da homeostase eletrolítica. Há quase 20 anos, essas substâncias desempenharam relevante papel na terapia da hipertensão arterial e nas doenças cardiovasculares. ${ }^{23,25}$

Essa classe de fármacos apresentou grande avanço desde que o primeiro composto, o captopril, foi liberado para uso clínico em 1981. Atualmente, existem no mercado em torno de 20 inibidores da enzima conversora de angiotensina (IECA) usados em vários países. ${ }^{25}$

Os inibidores da (ECA) impedem a conversão de angiotensina I em angiotensina II (fig. 4), que é um vasoconstritor potente, atenuando assim seus efeitos. ${ }^{19,23,25}$

O uso desse inibidor é a melhor alternativa de medicação por via oral ou sublingual para o tratamento de crises hipertensivas, especialmente aquelas em que o bloqueio do sistema reninaangiotensina-aldosterona (SRAA) tenha indicação preferencial, como na insuficiência cardíaca congestiva, acidente vascular cerebral, hipertensão arterial maligna e infarto agudo do miocárdio. ${ }^{26}$

Esta classe de anti-hipertensivo é formalmente contraindicada na gravidez e em estenose bilateral de artérias renais. Deve ter uso cauteloso em insuficiência renal, estados hipovolêmicos e em hipercalemia. ${ }^{19,26}$ Exemplos: enalapril, lisinopril, captopril.

Dentro desta classe incluem-se também os antagonistas dos receptores $\mathrm{AT}_{1}$ de angiotensina II, por exemplo, o losartan. ${ }^{18,27}$ Sabe-se que a angiotensina II liga-se a dois subtipos de receptores, $\mathrm{AT}_{1}$ e $\mathrm{o} \mathrm{AT}_{2}$, e todas as funções conhecidas da angiotensina II se dão pela ligação com os receptores $\mathrm{AT}_{1}$. Os antagonistas dos receptores da angiotensina II (AII) têm como mecanismo de ação o bloqueio das ações da AII pela ocupação específica do receptor $\mathrm{AT}_{1}$ exercendo consequentemente ação antihipertensiva e protetora para os diferentes órgãosalvo da hipertensão arterial. ${ }^{19}$ 


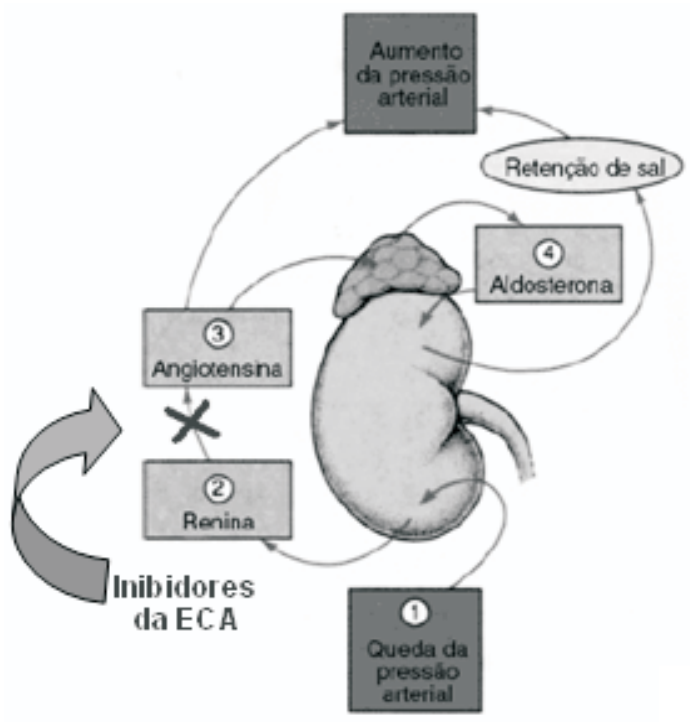

Figura 4 - Ação dos inibidores da enzima conversora de Angiotensina dos inibidores da enzima conversora de Angiotensinaconversora de angitensina normais, em $b$ mostra a frequncia(ECA) que impede a conversão de angiotensina I em angiotensina II. Adaptado do Portal da Educação, Curso de Fisiologia Geral, 2008.

\section{Outros mecanismos (Grupo V)}

Relaxante do músculo liso através do aumento seletivo da permeabilidade da membrana ao potássio, ocasionando hiperpolarização da membrana, desligando os canais de cálcio voltagem-dependentes e inibindo a geração de um potencial de ação (minoxidil). Existem também drogas que afetam a síntese da noradrenalina por ser um precursor de transmissor falso (metildopa). ${ }^{19}$

\section{MATERIAL E MÉTODOS}

O material para realização desta pesquisa foi obtido a partir da revisão bibliográfica de livros e artigos científicos publicados em periódicos localizados nas bases de dados Medline, Scielo e Lilacs, tendo como palavras-chave: hipertensão arterial sistêmica; anti-hipertensivos; idoso. Através das palavras-chave, localizaram-se 32 artigos, dos quais 20 foram utilizados para a pesquisa. Dos artigos selecionados, cinco são estudos sobre prevalência da HAS nos idosos em diferentes regiões, oito relacionados a fisiopatologia e diagnóstico da HAS no idoso e sete artigos sobre tratamento anti-hipertensivo.

Dos sete livros pesquisados, dois discorrem sobre a fisiopatologia da HAS e cinco trazem informações sobre o tratamento medicamentoso. Procurou-se excluir da pesquisa artigos e livros relacionados à hipertensão arterial no adulto, que tem outras particularidades no diagnóstico e terapêutica medicamentosa.

Trata-se de um estudo transversal com dados obtidos a partir de pesquisa em prontuários de anotações técnicas, para a caracterização dos quadros diagnosticados como hipertensão arterial, considerando anamnese, exame físico na admissão e controle dos níveis pressóricos diários dos pacientes da Unidade Terapêutica Estância do Instituto Bairral de Psiquiatria, entre os dias 18 a 25 de setembro de 2009 , com posterior análise das principais classes dos agentes antihipertensivos orais utilizados.

O critério utilizado para caracterização da hipertensão teve como base as $V$ Diretrizes Brasileiras 
de Hipertensão Arterial, que classificam como hipertenso o idoso com níveis pressóricos PAS $>140$ mmhg e PAD > 90mmhg.

A população analisada foi composta por 51 sujeitos com faixa etária entre 52 e 87 anos de idade, em sua maioria portadora de distúrbios psiquiátricos e outras comorbidades clínicas, sendo excluídos da amostra os pacientes sem histórico de HAS e com níveis pressóricos considerados normais pelas $V$ Diretrizes Brasileiras de Hipertensão Arterial.

O controle da pressão arterial dos pacientes analisados para complementação do diagnóstico da HAS foi realizado duas vezes ao dia, no período da manhã e tarde. Optou-se pela posição sentada, preconizando repouso prévio de 5 a 10 minutos, em ambiente confortável e calmo.

A finalização deste trabalho ocorreu com a comparação dos dados observados com os resultados presentes na literatura.

O projeto deste estudo foi aprovado pelo Comitê de Ética em Pesquisa da Faculdade de Ciências Médicas da UNICAMP (Parecer n.730/2009).

\section{RESULTADOS}

Dos 51 pacientes analisados no Setor Terapêutico Estância do Instituto Bairral de Psiquiatria, 27 foram diagnosticados com quadro de hipertensão Arterial no período em referência, correspondendo a $53 \%$ do número total da amostra. Utilizamos como parâmetro as $V$ Diretrizes Brasileiras de Hipertensão Arterial, que preconizam que as metas dos níveis pressóricos para idosos são PAS < $140 \mathrm{mmhg}$ e $\mathrm{PAD}<90 \mathrm{mmh}$.

Quanto à distribuição por sexo, houve predominância do sexo feminino com 15 casos, respondendo por $56 \%$ dos pacientes hipertensos para 12 casos do sexo masculino (44\%).

Quanto à frequência dos fármacos antihipertensivos prescritos nesta amostra, observouse que o grupo IV, pertencente ao grupo de fármacos que intervêm no sistema reninaangiotensina, foi o mais usado, com 19 prescrições (61,3\%), seguido pelo grupo III, dos diuréticos, com cinco prescrições (16,1\%), e os grupos I, II e $\mathrm{V}$, dos bloqueadores adrenérgicos, bloqueadores do canal de cálcio e outros, respectivamente, como o terceiro, quarto e quinto grupo de medicamentos mais usados no controle da hipertensão arterial.

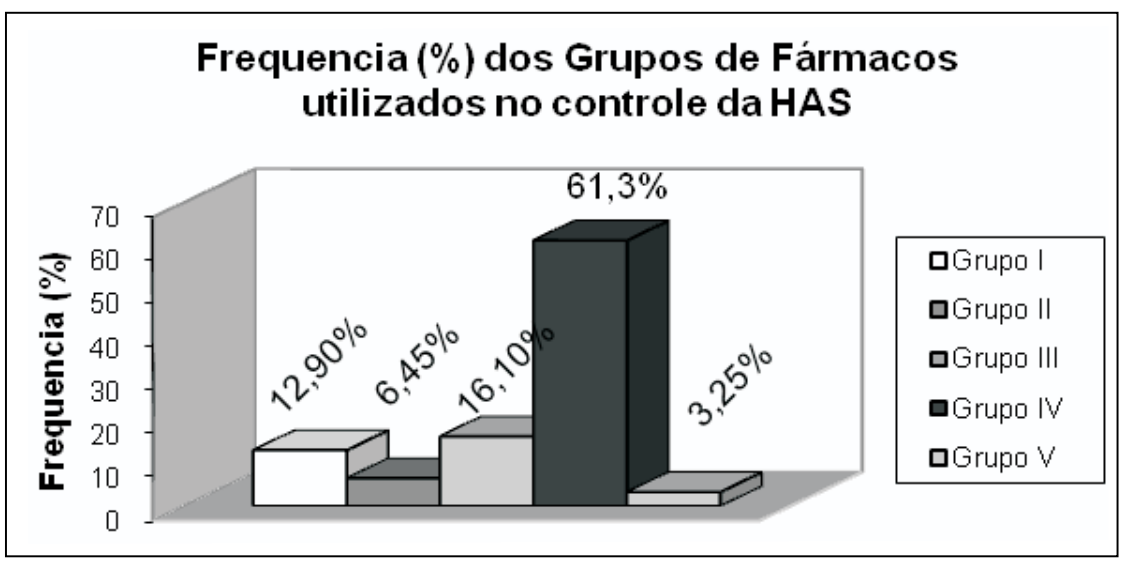

Figura 5 - Predominância de prescrições de fármacos. Itapira, SP, 2009. 
O bloqueio do sistema renina-angiotensinaaldosterona (SRAA) constitui uma excelente opção terapêutica para a hipertensão arterial (HA), bem como para a cárdio e nefroproteção. Dentro deste grupo, o fármaco mais prescrito no controle da pressão arterial foi o captopril, com oito pacientes em uso, correspondendo a $42,1 \%$, seguido pelo enalapril, com sete prescrições $(38,85 \%)$, e os demais com uma prescrição cada, respondendo por $5,26 \%$.

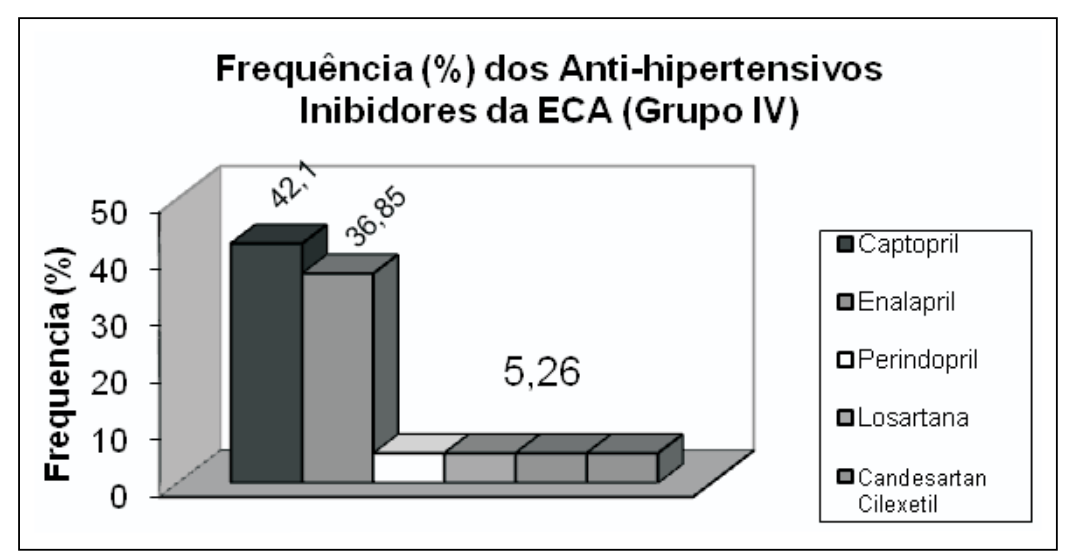

Figura 6 - Frequência em \% dos medicamentos que compõem o grupo IV (drogas que intervêm no sistema renina-angiotensina). Itapira, SP, 2009.

Paralelamente, o grupo III, que corresponde ao grupo dos diuréticos, foi a segunda classe de medicamentos mais utilizada para o controle da pressão arterial. Dentre os fármacos que se enquadram nesta classe, os diuréticos tiazídicos representados pela hidroclorotiazida e a clortalidona responderam por 60\% das prescrições (hidroclorotiazida 40\% e clortalidona $20 \%$ ), seguidos da furosemida, um diurético de alça (20\%), e a amilorida, um bloqueador dos canais de sódio dos túbulos coletores (20\%).

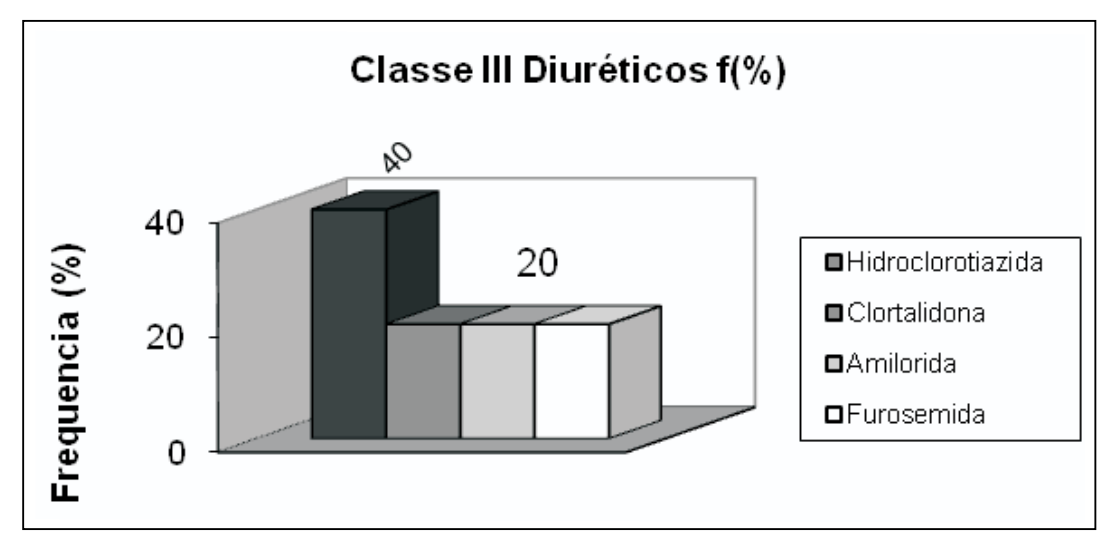

Figura 7 - Frequência em \% dos medicamentos que compõem o grupo III (diuréticos). Itapira, SP, 2009. 
Quanto às prescrições de fármacos antihipertensivos usados em associação, os inibidores da ECA, juntamente com os diuréticos, foram os mais prescritos, representando $50 \%$ das prescrições.

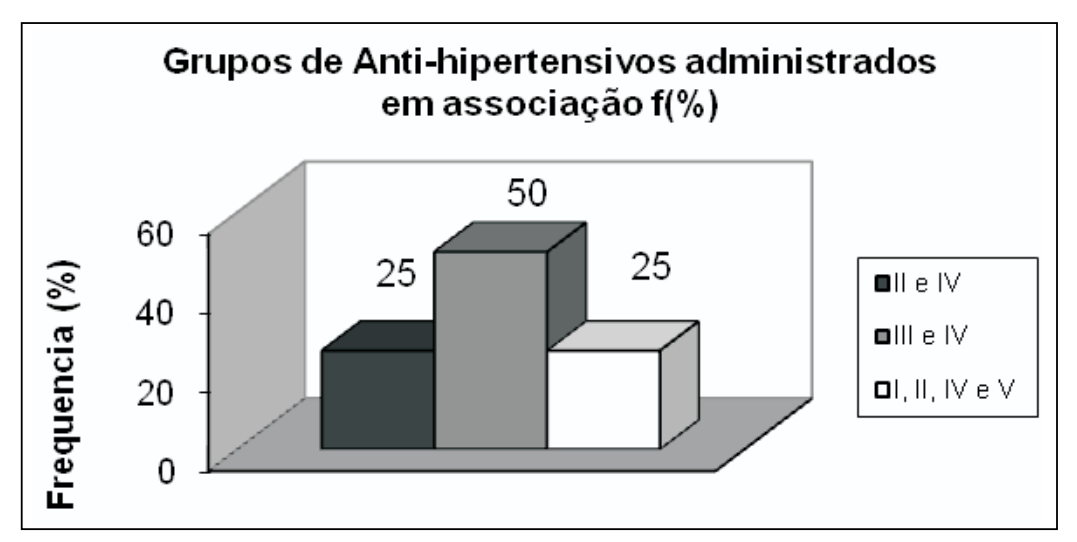

Figura 8 - Porcentagem dos fármacos administrados em associação. Itapira, SP, 2009.

Observou-se que uma parcela significativa da amostra - cinco casos $(18,52 \%)$ analisados apresenta o diagnóstico da HAS, porém não faz uso de medicamentos, apresentando como terapêutica a modificação do estilo de vida, com a realização de atividades físicas, redução do peso corporal e redução do sal na dieta.

\section{DISCUSSÃO}

Vários estudos demonstraram ser significativo o número da população idosa com HAS, analisando indivíduos de diferentes características sociais e diferentes regiões.

Em estudo realizado por Oliveira et al. ${ }^{6}$ com 1.265 mulheres idosas residentes no município deSão Paulo, encontrou-se prevalência de hipertensão arterial de $55,3 \%$ (60 a 74 anos) e $60,7 \%$ (75 anos ou mais).

Dados obtidos por Zaitune et $\mathrm{al} .{ }^{4}$ em estudo com 426 idosos na cidade de Campinas (SP) mostrou prevalência de HAS de $51,8 \%$, sendo $55,9 \%$ sexo feminino e $46,4 \%$ do sexo masculino.

Jobim \& Cabrera ${ }^{5}$ analisando 277 idosos cadastrados no Programa Saúde na Família do município de Jardim Alegre (PR), encontraram prevalência de HAS de $52 \%$, sendo $56 \%$ mulheres e $43 \%$ homens.
Outro estudo realizado por Azevedo $\& \mathrm{Paz}^{3}$ com 600 idosos atendidos no Centro de Convivência de Cuiabá (MT), apontou prevalência de HAS superior a $50 \%$ e, contrariando os demais estudos, nessa amostra o sexo masculino foi o mais acometido.

Converso \& Leocádio, ${ }^{2}$ em pesquisa realizada com 150 pacientes em dez Núcleos Municipais da Terceira Idade de Presidente Prudente (SP), encontraram prevalência de HAS $63,34 \%$, valor acima dos limites esperados. A porcentagem de homens e mulheres portadores de HAS nesse estudo foi semelhante.

Diante da prevalência de HAS tão elevada, tornam-se necessárias intervenções terapêuticas e educacionais que visem a reduzir possíveis eventos cardiovasculares entre os idosos. Ao longo dos anos, a terapia farmacológica anti-hipertensiva mostrou um grande impacto em termos de redução do risco para esses pacientes.

Quanto aos resultados obtidos, pode-se observar que os inibidores da ECA foi o grupo de medicamento mais prescrito na amostra, sendo o captopril o medicamento mais usado dentro deste grupo, seguido pelo enalapril.

Os inibidores da ECA são eficazes no controle anti-hipertensivo e diminuem os efeitos cardiovasculares em pacientes de alto risco. ${ }^{9}$ 
Segundo Rodrigues ${ }^{26}$, o captopril é a melhor alternativa de medicação por via oral ou sublingual para o tratamento de crises hipertensivas, especialmente aquelas em que o bloqueio do SRAA tenha indicação preferencial, como na insuficiência cardíaca congestiva, acidente vascular cerebral, hipertensão arterial maligna e infarto agudo do miocárdio.

Apesar de o captopril ser um medicamento frequentemente prescrito nas unidades de saúde, o enalapril apresenta-se consideravelmente como droga mais potente. Segundo Goodman \& Gilmam ${ }^{14}$, um ponto interessante é seu tempo de ação ser mais prolongado, sendo muito eficaz tanto em monoterapia como em associação com outras drogas, especialmente diuréticos.

As principais alterações adversas com o uso dessa classe de medicamentos são tosse seca, mudança no paladar e hipercalemia. Os bloqueadores do sistema renina e angiotensina 11 (BRA) têm eficácia semelhante aos IECAS, com menos efeitos adversos e melhor ajuste posológico. ${ }^{9}$

Quanto ao grupo dos diuréticos, Miranda et al. ${ }^{1}$ e Perroti et al., ${ }^{16}$ relatam que os diuréticos tiazídicos são os fármacos de primeira escolha como monoterapia nos idosos, exceto naqueles casos em que há uma indicação preferencial. Esses dados corroboram os achados da pesquisa, sendo que $60 \%$ dos diuréticos prescritos para este grupo de idosos eram diuréticos tiazidicos (hidroclorotiazida e clortalidona).

Apesar de alguns autores sugerirem que a clortalidona e a hidroclorotiazida (diuréticos tiazídicos) são equivalentes em propriedades farmacológicas, alguns estudos mostraram que a clortalidona parece ser mais potente que a hidroclorotiazida. ${ }^{21}$ Mesmo em baixas doses, os tiazídicos mantêm sua eficácia anti-hipertensiva, com baixo risco de efeitos colaterais (como hipopotassemia, hipomagnesemia e hiperuricemia), possuindo baixo custo e benefícios cardiovasculares comprovados. ${ }^{9,16}$

Apesar das evidências em favor da clortalidona, a hidroclorotiazida continua sendo o diurético tiazídico mais comum utilizado na prática clínica, dados também observados neste estudos uma vez que $40 \%$ dos diuréticos prescritos correspondem à hidroclorotiazida. Essa diferença ocorre sobretudo em relação ao reduzido número de combinações fixas contendo clortalidona, comparado com a grande disponibilidade de combinações fixas contendo hidroclorotiazida. No entanto, o médico precisa considerar as diferenças clínicas e farmacológicas ao escolher o diurético tiazídico mais apropriado. ${ }^{21}$

Os betabloqueadores e bloqueadores do canal de cálcio foram classes de medicamentos pouco prescritos na amostra analisada.

Segundo Gazoni et al. ${ }^{9}$ não é aconselhável utilizar betabloqueador como terapia inicial para tratamento da hipertensão arterial, pois não parece ser melhor do que outros agentes para prevenção primária de infarto agudo do miocárdio e acidente vascular cerebral. Está indicado somente para pósinfarto agudo do miocárdio e insuficiência cardíaca.

Já os bloqueadores do canal de cálcio (BBC) são anti-hipertensivos eficazes no tratamento da HSI do idoso, reduzem a incidência de AVC, porém pioram sintomas frequentes como constipação intestinal e edema de membros inferiores e aumento do volume urinário., ${ }^{1,9}$

Quanto às prescrições de fármacos antihipertensivos usados em associação, o estudo demonstrou que os inibidores da ECA, juntamente com os diuréticos, foram os mais prescritos, representando 50\% das prescrições.

Segundo Miranda et al. ${ }^{1}$ e Borelli et al., ${ }^{11}$ a terapia combinada com doses baixas de dois ou mais medicamentos reduz a pressão arterial de forma mais eficaz, com menos eventos adversos que a monoterapia em doses altas.

De acordo com Santello \& Mion $\mathrm{Jr}^{23}$ a associação de captopril à hidroclorotiazida um diurético tiazídico em dose baixa, oferece vantagens características do anti-hipertensivo ideal, como controle da pressão arterial (PA), redução da mortalidade cardiovascular, proteção cardíaca 
e renal, custo acessível e baixa incidência de efeitos colaterais.

Além do tratamento farmacológico da HAS, deve-se também envolver medidas nãofarmacológicas, como o aumento dos exercícios físicos, redução do sal das dietas e das gorduras saturadas com aumento de frutas e fibras, redução do peso corporal e do consumo de álcool e tabagismo.

Segundo Borelli et al., ${ }^{11}$ uma abordagem terapêutica nesse sentido pode trazer ganho adicional considerável ao tratamento do paciente hipertenso, melhorando até a resposta aos antihipertensivos utilizados.

\section{CONCLUSÃO}

Este estudo demonstrou ser significativo o número da população idosa com HAS no Setor Estância do Instituto Bairral de Psiquiatria, sugerindo acompanhamento clínico e medicamentoso dessa clientela.

A terapêutica medicamentosa adotada para tratamento da HAS no setor em questão está de acordo com a recomendada na literatura, bem como as medidas não-farmacológicas.

O tratamento da hipertensão arterial no idoso se faz com mudanças no estilo de vida, com realização de atividades físicas, modificações dos hábitos nutricionais e tratamento medicamentoso, exigindo dessa forma o acompanhamento de uma equipe multiprofissional.

O enfermeiro, como integrante da equipe multiprofissional, necessita obter maiores conhecimentos sobre a HAS e seu impacto na vida do idoso, a fim de planejar uma abordagem educativa.

Mais pesquisas nesse campo são necessárias para a obtenção de dados sobre prevalência da HAS no idoso e reconhecimento dos benefícios trazidos pelo tratamento anti-hipertensivo.

\section{REFERÊNCIAS}

1. Miranda RD, Perrotti TC, Bellinazzi VR, Nóbrega TM, Cendoroglo MS, Taniolo Neto J. Hipertensão arterial no idoso: peculiaridades na fisiopatologia, no diagnostico e no tratamento. Rev Bras Hipertens 2002; 9 (3) 293-300.

2. Converso MER, Leocádio PLLF. Prevalência da hipertensão arterial e análise de seus fatores de risco nos núcleos de terceira idade de Presidente Prudente. Rev Ciênc Extensão 2005; 2 (1)13.

3. Azevedo RG, Paz MAC. A prevalência de hipertensão arterial em idosos atendidos no centro de convivência para idosos em Cuiabá. Estud. Interdiscip. Envelhec 2006; 9: 101-115.

4. Zaitune MPA, Barros MBA, César CLG, Carandina L, Goldbaum M. Hipertensão arterial em idosos: prevalência, fatores associados, práticas de controle no município de Campinas. Cad Saúde Pública 2006; 22 (2): 285-294.

5. Jobim EFC, Cabrera MAS. Prevalência de hipertensão arterial em idosos atendidos no programa da saúde da família em Jardim Alegre, PR. Rev Envelhec Saúde 2007; 13 (2).
6. Oliveira SMJV, Santos JLF, Lebrão ML, Duarte YAO, Pierin AMG. Hipertensão arterial referida em mulheres idosas: prevalência e fatores associados. Rev Text Context Enferm 2008 abr-jun :17 (2): 241- 9.

7. Pimenta E, Calhoun DA. Uso dos antagonistas da aldosterona no tratamento da hipertensão arterial refratária. Rev Bras Hipertens 2007;14(4): 252-257.

8. Marte AP, Santos, RD. Bases fisiopatológicas da dislipidemia e hipertensão arterial. Rev Bras Hipertens 2008 ;14(4): 252-257.

9. Gazoni FM, Braga ILS, Guimarães HP, Lopes RD. Hipertensão sistólica no idoso. Rev Bras Hipertens 2009 ;16 (1): 34-37.

10. Jobim EFC. Hipertensão arterial no idoso: classificação e peculiaridades. Rev Bras Clin Méd 2008; 6: 250-253.

11. Borelli FA, Souza MG, Passarelli O, Pimenta E, Gonzaga C, Coredeiro A ,et al. Hipertensão arterial no idoso: importância em se tratar. Rev Bras Hipertens 2008; 15 (4): 236-239.

12. Sociedade Brasileira de Cardiologia; Sociedade Brasileira de Hipertensão. Diretrizes Brasileiras 
de Hipertensão Arterial .Sociedade Brasileira de Nefrologia; 2006.

13. Guyton AC, Hall JE. Tratado de Fisiologia Médica.11. ed. Rio de Janeiro: Elsevier; 2006.

14. Hardman JG, Limdbird LE .Goodman e Gilman: as bases farmacológicas da terapêutica. 8. ed. Rio de Janeiro: Guanabara Koogan; 1991.

15. Page CP. Farmacologia Integrada. São Paulo: Manole; 1999. p.178-190.

16. Perroti TC, Campos Filho J, Uehara CA, Almada Filho CM, Miranda RD. Tratamento farmacológico da hipertensão no idoso. Rev Bras Hipertens. 2007; 14(1): 37-41.

17. Rosa RF, Franken RA. Fisiopatologia e diagnóstico da hipertensão arterial no idoso: papel da monitorização ambulatorial da pressão arterial e da monitorização residencial da pressão arterial. Rev Bras Hipertens 2007 14(1): 21-24.

18. Prado FC, Ramos J, Valle JR. Atualização Terapêutica. 21 ed. São Paulo: Artes Médicas; 2003.

19. Rang HP, Dale MM, Ritter JM, Flower RJ. Rang e Dale Farmacologia. 6. ed. Rio de Janeiro: Elsevier; 2007.
20. Nigro D, Fortes ZB. Efeitos farmacológicos dos diuréticos e dos bloqueadores dos canais de cálcio Rev Bras Hipertens 2008; 12(2): 103-107.

21. Pimenta E. Hidroclorotiazida $x$ clortalidona: os diuréticos tiazídicos são todos iguais? Rev Bras Hipertens 2008; 15(3):166-167.

22. Henry JB. Diagnósticos clínicos e tratamento por métodos laboratoriais.18 ed. São paulo : Manole; 1995.

23. Santello JL, Mion D. Captopril Associado a Hidroclorotiazida no tratamento da hipertensão leve e moderada : estudo multicêntrico brasileiro . Arq Bras Cardiol 1998; 71(5) : 713-716.

24. Relman D. Tratado de Medicina Interna. 22 ed. Rio de Janeiro : Elsevier; 2005.

25. Ribeiro JM, Florêncio LP. Bloqueio farmacológico do sistema renina- angiotensinaaldosterona: inibição da enzima de conversão e antagonismo do receptor AT1.Rev Bras Hipertens 2000; 7(3).

26. Rodrigues CIS. Tratamento das emergências hipertensivas. Rev Bras Hipertens 1995 ; 9: 353 358.

27. Bicket DP. Using ACE inhibitors appropriately. Am Fam Physician 2002; 66 (3): 461. 